\title{
Detecting cell death with optical coherence tomography and envelope statistics
}

\author{
Golnaz Farhat, ${ }^{\mathrm{a}, \mathrm{b}, \mathrm{c}}$ Victor X. D. Yang, ${ }^{\mathrm{b}, \mathrm{d}, \mathrm{e}}$ Gregory J. Czarnota, ${ }^{\mathrm{a}, \mathrm{b}, \mathrm{f}, \mathrm{g}}$ and Michael C. Kolios ${ }^{\mathrm{a}, \mathrm{e}}$ \\ a University of Toronto, Department of Medical Biophysics, Toronto, Ontario, M5G 2M9, Canada

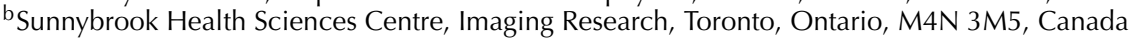 \\ cUniversity Health Network, Ontario Cancer Institute, Toronto, Ontario, M5G 2M9, Canada \\ ${ }^{\mathrm{d}}$ Ryerson University, Department of Electrical and Computer Engineering, Toronto, Ontario, M5B 2K3, Canada \\ e Ryerson University, Department of Physics, Toronto, Ontario, M5B 2K3, Canada \\ ${ }^{f}$ Sunnybrook Health Sciences Centre, Radiation Oncology, Toronto, Ontario, M4N 3M5, Canada \\ gUniversity of Toronto, Department of Radiation Oncology, Toronto, Ontario, M5S 3S2, Canada
}

\begin{abstract}
Currently no standard clinical or preclinical noninvasive method exists to monitor cell death based on morphological changes at the cellular level. In our past work we have demonstrated that quantitative high frequency ultrasound imaging can detect cell death in vitro and in vivo. In this study we apply quantitative methods previously used with high frequency ultrasound to optical coherence tomography (OCT) to detect cell death. The ultimate goal of this work is to use these methods for optically-based clinical and preclinical cancer treatment monitoring. Optical coherence tomography data were acquired from acute myeloid leukemia cells undergoing three modes of cell death. Significant increases in integrated backscatter were observed for cells undergoing apoptosis and mitotic arrest, while necrotic cells induced a decrease. These changes appear to be linked to structural changes observed in histology obtained from the cell samples. Signal envelope statistics were analyzed from fittings of the generalized gamma distribution to histograms of envelope intensities. The parameters from this distribution demonstrated sensitivities to morphological changes in the cell samples. These results indicate that OCT integrated backscatter and first order envelope statistics can be used to detect and potentially differentiate between modes of cell death in vitro. 02011 Society of Photo-Optical Instrumentation Engineers (SPIE). [DOI: 10.1117/1.3544543]
\end{abstract}

Keywords: optical coherence tomography; apoptosis; cell death; integrated backscatter; envelope statistics; treatment monitoring. Paper 10160RR received Mar. 24, 2010; revised manuscript received Sep. 29, 2010; accepted for publication Jan. 3, 2010; published online Feb. 17, 2011.

\section{Introduction}

Cancer therapies are aimed at stopping cell proliferation and/or inducing cell death. Typically, treatment response is evaluated based on changes in tumor size that may occur several weeks after the start of therapy. However, structural changes in responding cells can occur as early as $24 \mathrm{~h}$ after the initiation of treatment. Furthermore, it has been shown that early response to treatment is a good indicator of ultimate response for several types of cancer. ${ }^{1,2}$ Monitoring early response to treatment could be used to prevent the administration of ineffective treatments, thus reducing unnecessary side effects and potentially improving treatment outcomes for many patients. In preclinical research, treatment evaluation involves monitoring and quantifying cell death kinetics as they relate to a specific treatment. Currently, noninvasive research methods of measuring tumor response include small animal micro-computed tomography, high resolution magnetic resonance imaging and micro-positron emission tomography. ${ }^{3-5}$ These methods mainly rely on late changes in tumor size, vasculature, and tumor metabolic activity to draw conclusions on treatment efficacy. Detecting changes in cell structure related to cell death still requires invasive tissue analysis making longitudinal studies difficult and costly. Molecular imaging is also used to study the properties and effects of new

Address all correspondence to: Golnaz Farhat, Sunnybrook Health Sciences Centre, 2075 Bayview Avenue, Rm T2-167, Toronto, Ontario, M4N 3M5, Canada. Tel: 416-480-6100 ext. 83719; E-mail: golnaz.farhat@gmail.com. drugs $^{6}$ but requires the use of fluorescent or radionuclide labeled reporter probes, making this method expensive and time consuming. Furthermore, this technique does not yet translate well to the clinic. Currently, there is no standard noninvasive method to monitor cell death clinically or pre-clinically linked to morphological changes at the cellular level.

During cell death, characteristic structural transformations occur at the cellular level that can change the mechanical and optical properties of the cell. We have previously used quantitative spectroscopic methods with high frequency ultrasound imaging to detect cell death both in vitro and in vivo. In our past work, ultrasound measurements of cells undergoing apoptotic death showed a 6-fold increase in backscatter intensity compared to viable cells. ${ }^{7}$ Spectral analysis techniques developed by Lizzi et al. ${ }^{8}$ permitted the analysis of the frequency spectrum of backscattered ultrasound waves obtained from cell samples before and after treatment to induce apoptosis. Treated cell samples demonstrated changes in spectral parameters such as the spectral slope, $0 \mathrm{MHz}$ intercept, and the spectral midband fit compared to untreated cells. ${ }^{9}$ Envelope statistics of ultrasound signals have also been investigated by fitting the Rayleigh and generalized gamma probability distributions to a histogram of the signal envelope intensities. The fit parameters from these distributions have been demonstrated to be sensitive to morphological changes in cells. Furthermore, these

1083-3668/2011/16(2)/026017/7/\$25.00 @ 2011 SPIE 
parameters can be related to the size and number density of the scatterers based on certain assumptions about the size relative to the wavelength and distribution of the scatterers. Analysis of ultrasound backscatter signals obtained from mixtures of treated and untreated cells have demonstrated this method is capable of detecting a $2.5 \%$ apoptotic index under well controlled conditions. ${ }^{10}$

Light scattering has a long standing history in oncological research. Spectroscopic analysis of backscattered light can be used to differentiate between dysplastic and nondysplastic epithelial cells. Light scattering spectroscopy has been used in vivo to diagnose dysplasia by determining nuclear size distribution ${ }^{11}$ and the spectroscopic analysis of optical coherence tomography (OCT) images has been used for differentiating between cell types based on cell and nuclear morphology. ${ }^{12}$ Recently, variations in the optical attenuation coefficient of human fibroblast cell samples have been detected as a result of cell death in vitro. ${ }^{13}$ Changes in relative tissue scattering have also been detected between viable and nonviable tissue regions in a mouse tumor model using optical frequency domain imaging. ${ }^{14}$ Optical coherence tomography and high frequency ultrasound share many similarities in manners of data acquisition and image formation, for which we previously conducted a feasibility study in monitoring treatment responses. ${ }^{15}$ Based on these similarities, here we have applied techniques used previously for cell death monitoring to OCT.

The work described here tests the hypothesis that structural changes occurring within dying cells will induce observable changes in measured OCT backscatter signals. The in vitro biological models used in our experiments are comprised of acute myeloid leukemia (AML) cells centrifuged to mimic cell packing observed in mouse tumor xenograft models. This tumor model has been extensively used for both single time point and time course studies of cell death and AML cell death has been well characterized histologically in our laboratory. ${ }^{7,9,16,17}$ For the study here three types of cell death were induced in these cells: apoptosis, mitotic arrest, and necrosis. Morphologically, apoptosis is characterized by nuclear condensation and fragmentation, followed by blebbing of the cell membrane and disintegration of the cell into apoptotic bodies composed of intact organelles surrounded by a plasma membrane. Recently it has been demonstrated that changes in mitochondrial morphology may also be associated with apoptosis, such as the disintegration of well formed mitochondrial networks into punctiform, fragmented structures. ${ }^{18}$ Cells arrested in mitosis are slightly enlarged and show increased chromatin content due to the condensation and doubling of chromosomes in preparation for cell division. Necrosis, caused by a failure in the ionic pumps of the cellular membrane due to a lack of adenosine triphosphate (ATP), is characterized by initial cellular and organelle swelling (oncosis) followed by budding of the cell membrane and cellular lysis. ${ }^{19,20}$

In this study we demonstrate that cell death can be detected by optical coherence tomography. Different modes of cell death were induced in cell samples and imaged using OCT. We demonstrate that an increase in backscatter intensity is associated with apoptosis and mitotic arrest and a drop in intensity with necrosis. The research here in vitro forms a basis for future investigations in vivo using optical methods to monitor the cell death causing targets of anti-cancer therapies.

\section{Materials and Methods}

\subsection{Biological Samples}

Cell samples were prepared using acute myeloid leukemia cells (AML-5). This cell line permits the rapid production of large numbers of cells for our experiments and has previously been characterized using high frequency ultrasound in our laboratory. Approximately $1 \times 10^{9}$ AML cells started from frozen stock samples were grown at $37^{\circ} \mathrm{C}$ in suspension flasks containing $150 \mathrm{~mL}$ of $\alpha$-minimal medium supplemented with $1 \%$ streptomycin and $5 \%$ fetal bovine serum. Each cell sample was prepared from four flasks.

Apoptosis was induced by treating the cells with the chemotherapeutic agent cisplatin, a DNA intercalater that causes a p53-dependent apoptosis. ${ }^{21}$ Cells were treated in suspension at $10 \mu \mathrm{g} / \mathrm{mL}$ for $24 \mathrm{~h} .{ }^{17}$ Mitotic arrest was induced in a second batch of cells using colchicine at $0.1 \mu \mathrm{g} / \mathrm{mL}$ for $24 \mathrm{~h}$ prior to cells being processed for imaging. ${ }^{7}$ This drug inhibits the formation of tubulin, causing the arrest of cells at metaphase in the mitotic cycle. ${ }^{22}$ Cells were washed with phosphate buffered saline (PBS) and centrifuged in a bench top swing bucket centrifuge (Jouan CR4i, Thermo Fisher Scientific, Waltham, MA) in a flat bottom ependorff tube at $2000 \mathrm{~g}$ producing a densely packed cell sample approximately $1 \mathrm{~cm}$ in diameter and $0.5 \mathrm{~cm}$ in height. The supernatant above the cell pellet was carefully removed using a $200 \mu \mathrm{L}$ pipette tip. Necrotic cell death was induced by growth factor withdrawal by maintaining an untreated centrifuged cell sample in PBS at room temperature for $24 \mathrm{~h}$. The absence of growth medium during this period results in a lack of ATP produced by the cells, which, in turn, induces ionic pump failure in the cell membrane, leading to oncosis and eventually necrosis ${ }^{20}$ and was histologically confirmed. Trypan blue exclusion confirmed cell death in approximately 80,60, and $25 \%$ of cells treated with cisplatin, colchicine, and growth factor withdrawal, respectively. For each experiment an additional cell sample was produced from untreated cells to serve as a control. Although every effort is made to ensure that cell culture is done under very controlled conditions, variations in the cell samples arise between different batches of cells or from harvesting cells at different passage numbers and phases of growth. For this reason, each experiment is conducted with a control sample obtained from the same batch under the exact same growth conditions.

\subsection{Reference Phantom}

An optical scattering phantom was used as a reference for normalizing all signals measured from cell samples to maintain consistency between experiments and to remove systematic errors. The use of a reference phantom is common practice in ultrasound tissue characterization experiments, where a flat quartz is used to obtain a calibration spectrum. ${ }^{8}$ The optical phantom was prepared from a silicon base mixed with titanium dioxide as the scattering agent, at a concentration of $0.1 \mathrm{~g} / \mathrm{mL}$. Reference measurements were collected at each data acquisition to later remove system effects.

\subsection{Histology and Light Microscopy}

Immediately after the completion of OCT imaging, cell samples were fixed in $10 \%$ formalin for $48 \mathrm{~h}$ and subsequently paraffin 


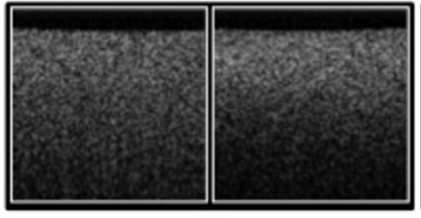

(a)

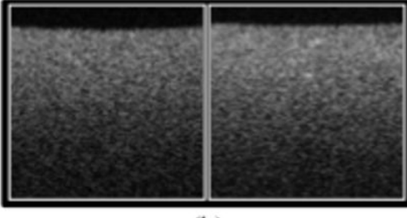

(b)

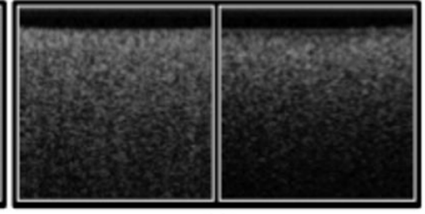

(c)

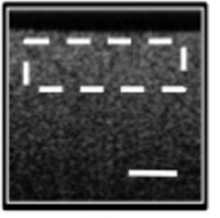

(d)

Fig. 1 Optical coherence tomography b-mode images of centrifuged cell samples. In each of the first three panels the control sample is shown on the left and the treated sample on the right. (a) $24 \mathrm{~h}$ cisplatin treatment, (b) $24 \mathrm{~h}$ colchicine treatment, and (c) $24 \mathrm{~h}$ growth factor withdrawal. The region of interest (measuring $600 \mu \mathrm{m}$ by $250 \mu \mathrm{m}$ ) used for quantitative analysis is outlined by the white dotted frame in an example image (d). Scale bar indicates $250 \mu \mathrm{m}$.

embedded and processed for haematoxylin and eosin $(\mathrm{H} \& \mathrm{E})$ staining. Microscopy was carried out using a Leica DM LB microscope and digital images were acquired with the Leica DC 200 digital imaging system (Leica Microsystems GmbH, Germany).

\subsection{OCT Data Acquisition and Analysis}

Optical coherence tomography images and data were acquired using a Thorlabs Inc. (Newton, NJ) swept source OCT (OCM1300SS) system. This system uses a frequency swept external cavity laser with a central wavelength of $1325 \mathrm{~nm}$ and a $-3 \mathrm{~dB}$ bandwidth of approximately $100 \mathrm{~nm}$ with an axial resolution of $9 \mu \mathrm{m}$. The laser is coupled to a fiber-based Michelson interferometer and is split to reference and sample arms using a broadband 50/50 coupler. The mean beam spot size in the focal plane is $15 \mu \mathrm{m}$ and the average output power of the system is $10 \mathrm{~mW}$.

Data were acquired using the Thorlabs Swept Source Optical Coherence Tomography Microscope software package (version 1.3.0.0, Thorlabs Inc.) and data were collected in the form of 14-bit OCT interference fringe signals. For each cell sample (including the reference phantom) two dimensional (2D) data sets containing 512 axial scans were collected in ten planes spaced at least $10 \mu \mathrm{m}$ apart. Each 2D scan covered a total transverse distance of $3 \mathrm{~mm}$ and a depth of $3 \mathrm{~mm}$. All samples (including the reference phantom) were imaged such that the surface was located at the same distance in relation to the focal plane to maintain consistency between measurements with respect to variations in signal intensity due to the focal properties of the lens and the depth dependent signal roll-off of the laser source.

As shown in Fig. 1(d), from each 2D scan a region of interest (ROI) covering a depth of $250 \mu \mathrm{m}$ and a lateral distance of $600 \mu \mathrm{m}$ was selected for analysis. These distances corresponded to 190 and 460 times the central wavelength, respectively. The lateral dimension was chosen to give approximately 100 a-lines per b-mode image. The axial dimension was chosen to coincide with the depth of focus of the system and begins at approximately $30 \mu \mathrm{m}$ below the cell sample surface to avoid any specular reflections.

Normalized backscatter power spectra were obtained from individual a-lines by calculating the Fourier transform of the corresponding complex interference signals and normalizing the squared magnitude of these spectra by the power spectrum computed from the titanium dioxide reference phantom. The resulting normalized power spectra were individually integrated over the $-6 \mathrm{~dB}$ bandwidth of the light source to calculate the integrated backscatter (IB). An average IB value was computed for each 2D ROI. For the first order envelope statistical analysis, histograms were calculated from the magnitude of the envelopes of acquired interference signals for each 2D ROI. These histograms were calculated with the goal of looking at the general statistics of the intensity distribution. Since the drop in intensity over $250 \mu \mathrm{m}$ is small, attenuation calculations and corrections were not performed. Generalized gamma parameters were obtained for the histograms using an implementation of a maximum likelihood estimation routine. ${ }^{10}$ A set of average parameters was calculated for each 2D ROI. The estimated error was calculated as a standard deviation of each of the above parameters over the ten ROIs acquired. A representative plot of the estimated error as a function of the number of a-lines averaged per ROI (Fig. 2) indicates that the uncertainty in the results stabilizes above 20 a-lines. The 100 a-lines per ROI used in our analysis are, therefore, deemed sufficient to ensure reliability of our results. The statistical significance for all parameters was determined by performing a student t-test using the GraphPad Prism software package (GraphPad Software Inc., La Jolia, CA), where $\mathrm{p}<0.05$.

\section{Results}

Optical coherence tomography b-mode images obtained from the cell samples are shown in Fig. 1, where changes in backscatter intensity were observed between the control and treated samples from each experiment. Backscatter power spectra (Fig. 3) for all three experiments indicated changes between the

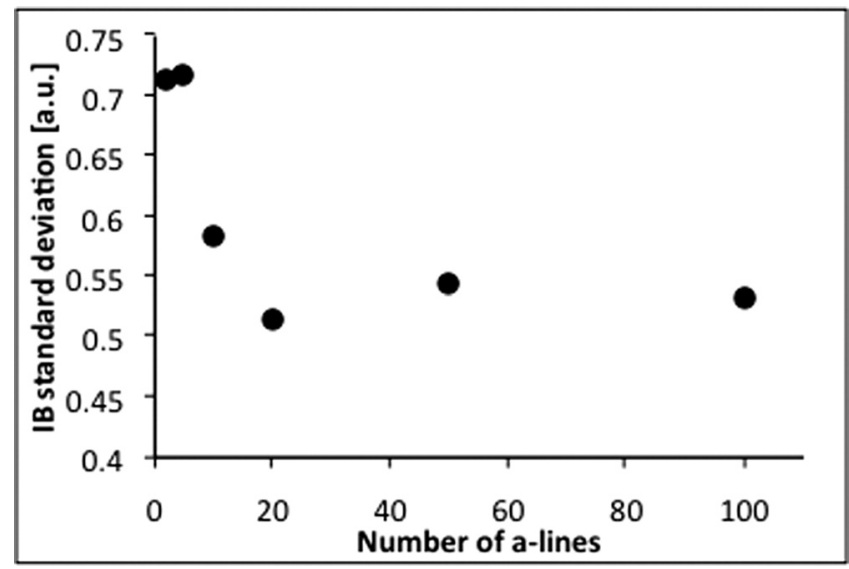

Fig. 2 Standard deviation for the integrated backscatter measurement from ten ROIs as a function of the number of a-lines averaged per ROI. Above 20 a-lines the standard deviation stabilizes. 


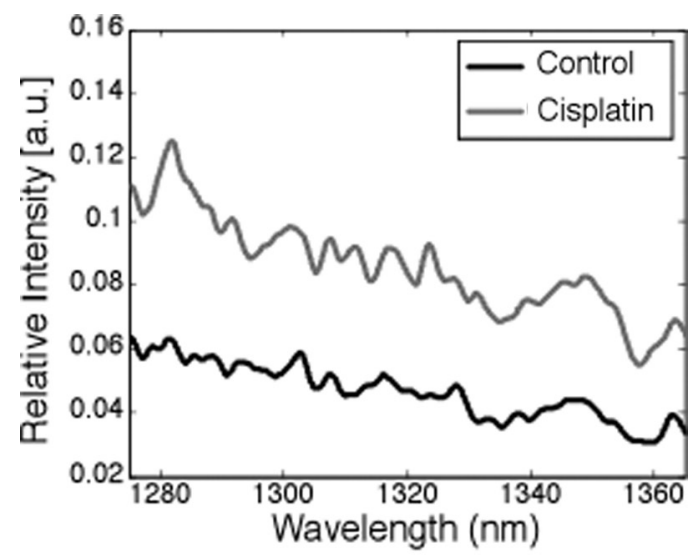

(a)

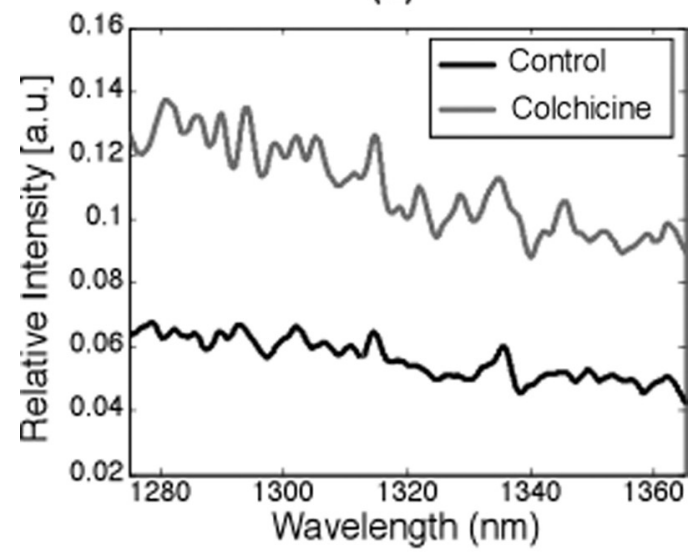

(b)

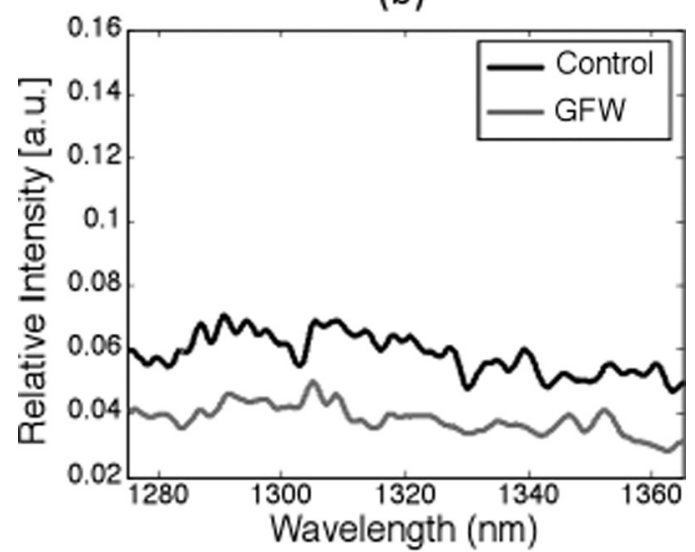

(c)

Fig. 3 Measured relative backscatter power for (a) cisplatin, (b) colchicine, and (c) growth factor withdrawal experiments. Backscatter power normalized to the reference phantom is plotted as a function of wavelength for control (untreated) cells and treated cells for each experiment. These results represent the average backscatter power measured from ten ROls for each sample.

control and treated samples, with increases in backscatter intensity observed for the cisplatin and colchicine treated cells (approximately 80 and 95\%, respectively) and a decrease (35\%) for the cells decaying in PBS. Integrated backscatter is related to backscatter intensity and the graphs in Fig. 4 indicate significant $(\mathrm{p}<0.05)$ changes with backscatter increasing from $4.5 \pm 0.4$ to $8.2 \pm 1.0$ with cisplatin exposure, increasing from $5.0 \pm 0.6$ to $9.8 \pm 1.3$ with colchicine treatment, and decreas-

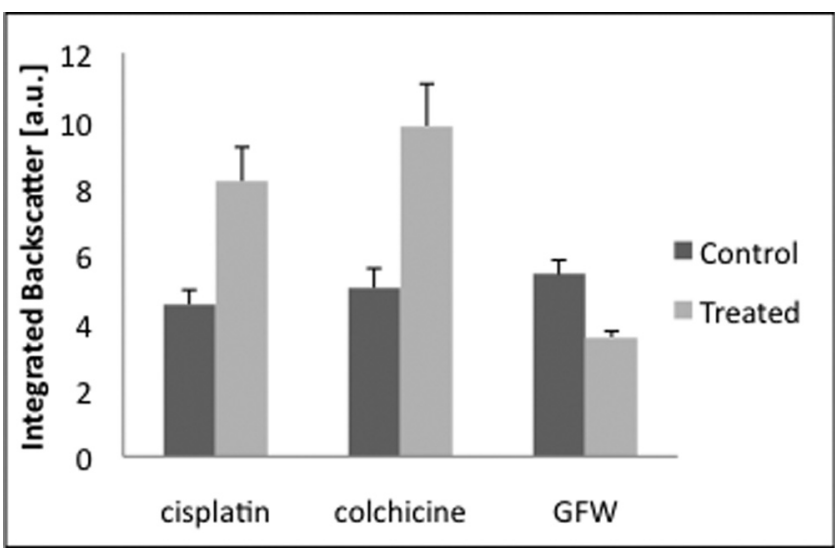

Fig. 4 Integrated backscatter measurements for cisplatin, colchine, and growth factor withdrawal experiments. Values shown were obtained by integrating the backscatter power over the $-6 \mathrm{~dB}$ bandwidth of the OCT system light source and averaging the result over the ten regions of interest measured for each sample. Error bars correspond to the standard deviation between the average integrated backscatter values for ten ROIs.

ing from $5.4 \pm 0.4$ to $3.6 \pm 0.2$ for the cells undergoing growth factor withdrawal.

Plots of generalized gamma fits to histograms from all three experiments (Fig. 5) demonstrate that this probability density function provides a reasonable fit to histograms calculated from OCT signal envelopes obtained from cell samples. Parameters from the generalized gamma fits are shown in Fig. 6. The average generalized gamma $a$ parameter did not significantly change $(\mathrm{p}=0.15)$ with cisplatin treatment (changing from $0.28 \pm 0.03$ to $0.32 \pm 0.06$ ), but increased from $0.26 \pm 0.03$ to $0.33 \pm 0.04$ for cells exposed to colchicine and decreased from $0.18 \pm 0.03$ to $0.09 \pm 0.02$ for cells withdrawn from growth medium. The $c / v$ ratio decreased from $1.31 \pm 0.29$ to 0.94 \pm 0.23 with cisplatin treatment and from $0.55 \pm 0.09$ to $0.34 \pm 0.09$ for cells undergoing growth factor withdrawal while the change with colchicine exposure $(1.1 \pm 0.24$ to 0.95 $\pm 0.20)$ was not significant $(\mathrm{p}=0.14)$.

Microscopy and analysis of haematoxylin and eosin (H\&E) stained fixed cells revealed structural differences between the various cell samples (Fig. 7). In the control sample, the cells appear viable, with a large nucleus occupying the majority of the cell volume, typical of AML cells. The cisplatin treated sample displayed classic signs of apoptosis with nuclear condensation and fragmentation in approximately $40 \%$ of cells. The most striking changes were detected in the colchicine treated cells. Here we observed $55 \%$ of cells in mitotic arrest with large amounts of highly condensed chromatin within the cell. The main feature to be observed in the cells deprived of growth medium was the condensation of the nucleus in roughly $40 \%$ of the cells. It must be noted that H\&E staining and light microscopy are limited in their ability to reveal all of the structural changes that may have occurred within these cells as a result of the treatments they underwent, particularly to smaller structures, such as the mitochondria.

\section{Discussion and Conclusions}

This study has demonstrated that it is possible to detect cell death in vitro using OCT. The changes in integrated backscatter power 


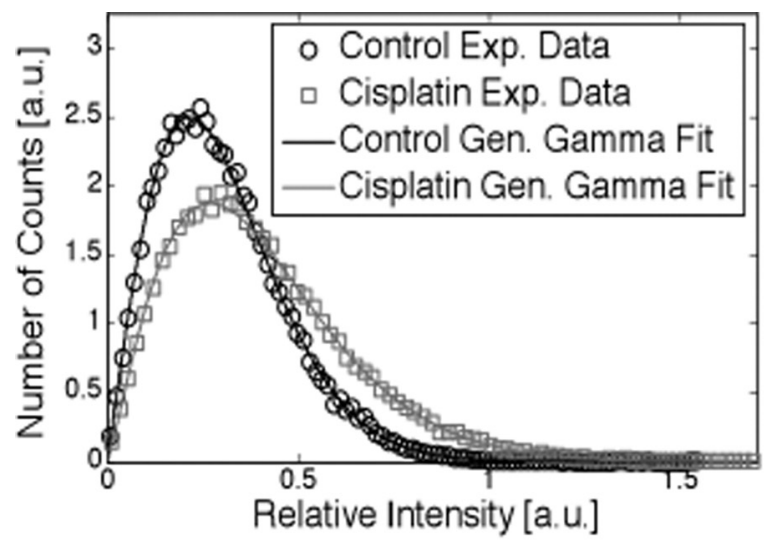

(a)

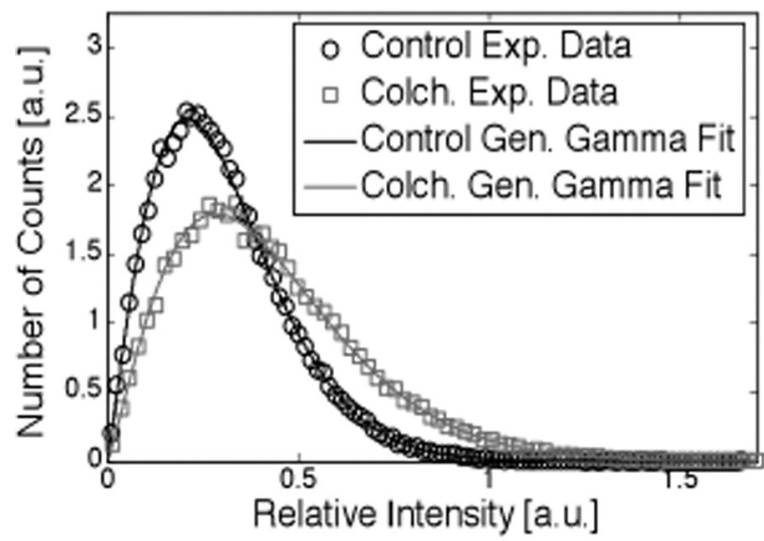

(b)

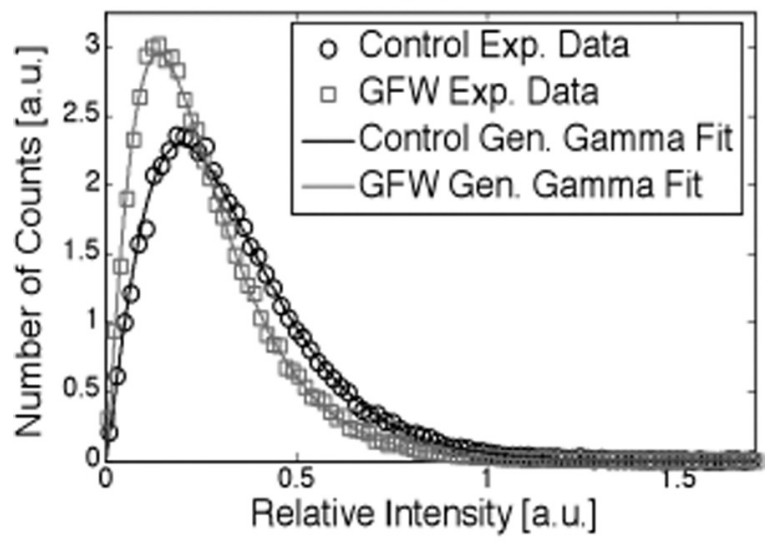

(c)

Fig. 5 Histograms of the amplitude of the envelope of OCT signals acquired from control and treated cell samples and corresponding fits of the generalized gamma distribution. The changes in scale are related to changes in backscatter intensity and correlate with changes in integrated backscatter seen in Fig. 4.

spectra were correlated with structural changes in histology, which from observations were grossly related to the nucleus and overall cell shape. The generalized gamma distribution provided reasonable fits to all cell sample histograms. As the scale parameter, $a$, increases, the distribution becomes wider, stretching along the intensity axis of the histogram. A change in the ratio of the shape parameters $(c / v)$ causes the center of the distribution to

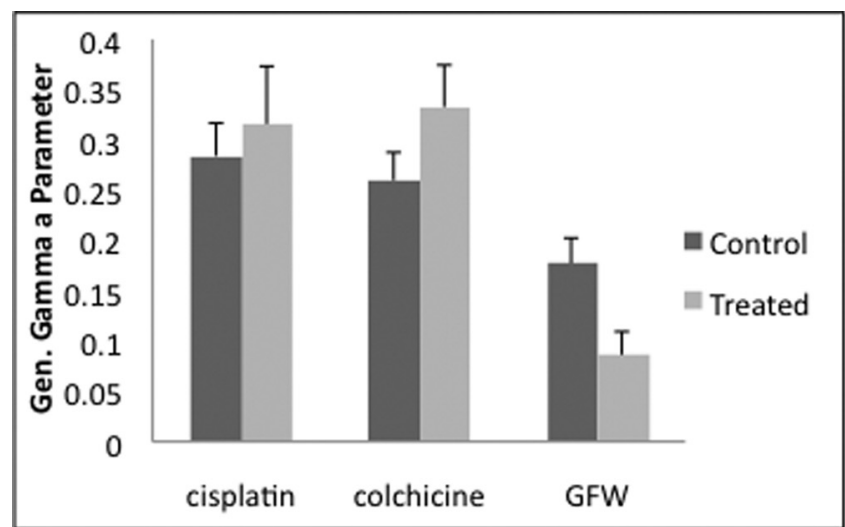

(a)

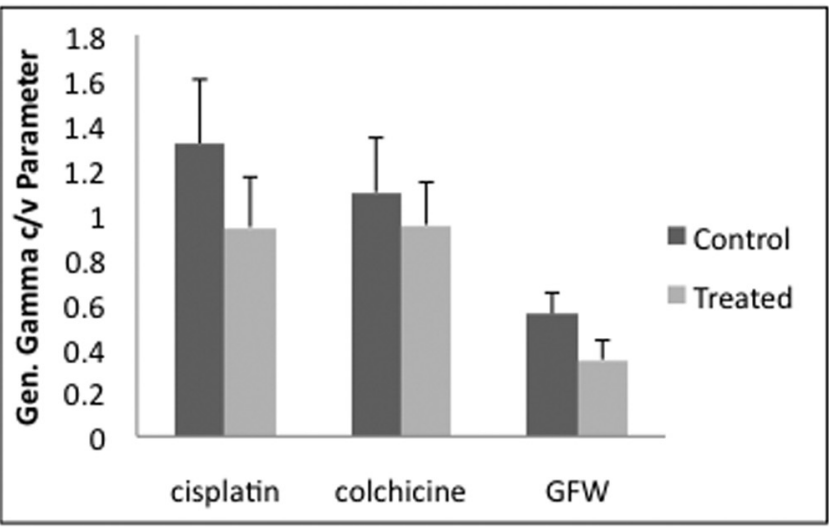

(b)

Fig. 6 Generalized gamma a parameter (a) and $c / v$ ratio (b). The a parameter is the scale parameter of the generalized gamma distribution and is related to backscatter intensity. The $c$ and $v$ parameters are the shape parameters of the generalized gamma distribution. It is suggested that their ratio can be related to the scatterer number density. Error bars correspond to the standard deviation between the average parameters obtained over ten ROls in a given cell sample.

shift along the intensity axis in the direction of the change. It has been suggested for ultrasound data that the generalized gamma $a$ parameter is related to the backscatter cross-section and that the ratio of the two parameters $c$ and $v$ can be used as an estimate of the relative scatterer number density. ${ }^{10,23}$ In this study the $a$ parameter paralleled, as expected, the integrated backscatter for all cell samples, though the change was not statistically significant for the apoptotic sample. The ratio of the shape parameters $(c / v)$ decreased for the apoptotic and necrotic cells and remained constant for the cells in mitotic arrest. The relationship between the $c / v$ ratio and scatterer number density was proposed based on ultrasound simulations and phantom experiments, ${ }^{23}$ and in vitro high frequency ultrasound experiments using cells in solution at low volumetric concentrations. ${ }^{10}$ It is not clear which structural changes are specifically responsible for a decrease in scatterer number density for OCT measurements from apoptotic and necrotic cells. However, ultrasound simulations have shown that even when the scatterer number density remains constant, changes in the randomization of the spatial distribution of scatterers can have an effect on this ratio. ${ }^{10}$ Given the variations that may exist between batches of cells it is not unexpected to observe differences between the parameters obtained from independent untreated samples. Furthermore, the scale parameter, 

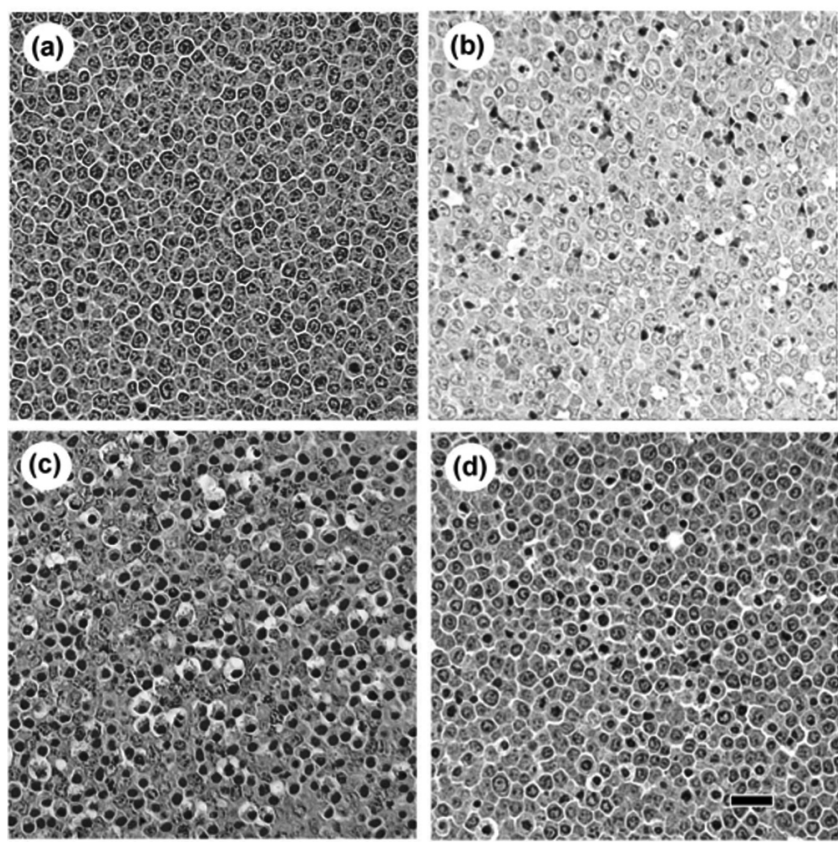

Fig. 7 Haematoxylin and eosin stained sections obtained from (a) untreated (viable) cells, (b) cells treated for $24 \mathrm{~h}$ with cisplatin (apoptotic), (c) cells treated for $24 \mathrm{~h}$ with colchicine (cells in mitotic arrest), and (d) cells withdrawn from growth medium for $24 \mathrm{~h}$ (oncotic/necrotic). Viable cells are circular in shape with nuclei that occupy the majority of the cell volume. Apoptotic cells exhibit pyknotic nuclei that are condensed as well as nuclear fragmentation. Cells undergoing blebbing of the membrane are also present. The white spacing is a retraction artifact typical of apoptotic samples. The mitotically arrested cells show an increase in chromatin that is condensed (dark blue staining). The oncotic/necrotic cell sample has cells with condensed nuclei but no nuclear fragmentation. Average cell/nuclear diameters measured from images in $\mu \mathrm{m}$ were: (a) $8.4 \pm 0.8 / 6.6 \pm 0.8$, (b) $8.2 \pm 1.3 / 4.2 \pm 1.9$, (c) $9.6 \pm 1.5 / 4.7 \pm 0.8$, and (d) $8.9 \pm 1.2 / 5.3 \pm 1.4$. Scale bar indicates $20 \mu \mathrm{m}$.

$a$, and the ratio of the shape parameters, $c / v$, are not independent, as a variation in the shape of the distribution will certainly cause changes to the scale of the distribution. Therefore, these parameters should not be individually considered, but rather as a multiparametric measurement, which can be related to cell death.

Previous experiments with the same cell line and treatments using high frequency ultrasound have resulted in increases in ultrasound backscatter intensity for apoptosis and mitotic arrest and a decrease for necrosis at $24 \mathrm{~h}$. Here, using OCT, there was an increase in backscatter for the apoptotic and mitotically arrested samples and a decrease in backscatter for the necrotic sample $24 \mathrm{~h}$ after treatment. Changes in ultrasound backscatter have been related to variations in nuclear structure. With OCT it remains unclear which cellular structures are responsible for the dominant fluctuations in backscatter observed during cell death. However, the current results indicate the potential for differentiating between apoptotic and oncotic or necrotic cell death using OCT.

Although nuclear changes in the growth factor withdrawn cell sample are not as large as those seen in the colchicine treated cells, the change in integrated backscatter and gamma $a$ parameter are significant in both. This leads us to believe that in addition to nuclear variations, there are other underlying struc- tural transformations within the cells contributing to measured changes in OCT backscatter. Further exploration of a relation to alterations in smaller cellular structures remains to be made.

The $24 \mathrm{~h}$ imaging time for cisplatin treatment was chosen in part based on our experience with ultrasound imaging of these cells, where cisplatin-induced apoptosis of AML cells produced maximal scattering changes after approximately $24 \mathrm{~h}$ of treatment. Maintaining the same imaging schedule for all modes of cell death permitted a direct comparison between cells undergoing apoptosis, necrosis, and mitotic arrest. In a study by van der Meer et al., human fibroblast cells treated in vitro with cytosine arabinoside (AraC) produced a maximal change in the optical attenuation coefficient $3 \mathrm{~h}$ post treatment, decreasing thereafter. ${ }^{13}$ In that study, AraC was believed to cause apoptosis through oxidative stress, a much more rapid pathway compared to apoptosis induction through DNA intercalation, which is what occurs with cisplatin. Cisplatin-induced apoptosis is highly dependent on the rate of cell growth and cell division. Thus, one cannot assume that both cell death models have the same temporal dynamics with respect to changes in optical properties caused by apoptosis.

In the same study, van der Meer et al. measured increases and decreases in optical attenuation for apoptotic and necrotic cells, respectively. Attenuation of light in tissue is caused by a combination of absorption and scattering. In the near infrared region, scattering is the dominant interaction of light with biological tissue and overall attenuation is significantly lower at $1300 \mathrm{~nm}$ compared to $800 \mathrm{~nm} .^{24}$ Assuming that the absorption remains constant, an increase in backscatter will result in an overall increase in attenuation while a decrease in backscatter will result in an overall decrease in attenuation, suggesting that our results agree in principle with those of the human fibroblast study. Our current technique may provide complementary information to the attenuation coefficient measurement, which could permit better quantification of cell death.

The in vitro model used in this work readily lends itself to further characterization of cell death using OCT. This model can be used for experiments in which dosage, timing, and the percentage of cells treated can be rigorously controlled. The cell death process has complex temporal dynamics that can significantly vary based on the length of time in culture and the stage of the cell growth cycle. Even in a well controlled in vitro study these factors will vary from one batch of cells to another, highlighting the fact that one must exercise caution in interpreting data obtained from in vivo experiments where the microenvironment is less well controlled. In these experiments we measured changes at the $24 \mathrm{~h}$ time point. The degree of OCT signal changes as well as the direction in trends observed may be different at earlier and later times. The sensitivity of this method with respect to quantifying cell death has not been tested. This can be achieved by imaging cell samples containing a mixture of treated and untreated cells at varying ratios ${ }^{10}$ and will be the subject of future work. The data acquisition and analysis methods described in this paper allow us to obtain backscatter spectra from specific regions of interest in measured cell samples in a reliable and reproducible manner. Although changes in spectral features were not measured in this study we believe that using this technique at shorter wavelengths or with larger bandwidths would allow for further spectroscopic analysis and will be explored in future studies. 
This is the first time, to our knowledge, that integrated backscatter spectra and first order envelope statistics have been used to detect cell death with OCT in vitro. Future work will involve the use of these methods for the characterization of cell death with OCT as well as the further development of these tools for cell death quantification.

\section{Acknowledgments}

The authors would like to thank Lilian Doss, Anoja Giles, and Dr. Adrian Mariampillai for technical support. The research here was supported by grants from the Natural Sciences and Engineering Council of Canada, the Canadian Institutes of Health Research, the American Institute of Ultrasound in Medicine, and the Terry Fox Foundation. Infrastructure support was provided by grants from the Canadian Foundation for Innovation. Additional funding was provided by the Canada Research Chairs program with awards to Dr. Victor Yang (Bioengineering and Biophotonics) and Dr. Michael Kolios (Biomedical Applications of Ultrasound), as well as the Cancer Care Ontario Research Chairs program with an award to Dr. Czarnota (Experimental Therapeutics and Imaging).

\section{References}

1. F. J. Esteva and G. N. Hortobagyi, "Can early response assessment guide neoadjuvant chemotherapy in early-stage breast cancer?," J. Natn. Cancer Inst. 100, 521 (2008).

2. M. A. Pantaleo, M. Nannini, A. Maleddu, S. Fanti, V. Ambrosini, C. Nanni, S. Boschi, and G. Biasco, "Conventional and novel PET tracers for imaging in oncology in the era of molecular therapy," Cancer Treat Rev. 34 103-121 (2008).

3. M. J. Paulus, S. S. Gleason, M. E. Easterly, and C. J. Foltz, "High resolution $\mathrm{X}$-ray computed tomography: an emerging tool for small animal cancer research," Neoplasia 2(1-2), 62-70 (2001).

4. J. S. Lewis, J. M. Connett, J. R. Garbow, T. L. Buettner, Y. Fujibayashi, J. W. Fleshman, and M. J. Welch, "Copper-64-pyruvaldehyde-bis(N(4)methylthiosemicarbazone) for the prevention of tumor growth at wound sites following laparoscopic surgery: monitoring therapy response with microPET and magnetic resonance imaging," Cancer Res. 62, 445-449 (2002).

5. Z. He, J. L. Evelhoch, R. M. Mohammad, N. V. Adsay, G. R. Pettit, V. K. Vaitkevicius, and F. H. Sarkar, "Magnetic resonance imaging to measure therapeutic response using an orthotopic model of human pancreatic cancer," Pancreas 21, 69-76 (2000).

6. J. K. Willmann, N. van Bruggen, L. M. Dinkelborg, and S. S. Gambhir, "Molecular imaging in drug development," Nat. Rev. Drug Discovery 7, 591-607 (2008).

7. G. J. Czarnota, M. C. Kolios, J. Abraham, M. Portnoy, F. P. Ottensmeyer, J. W. Hunt, and M. D. Sherar, "Ultrasound imaging of apoptosis: high-resolution non-invasive monitoring of programmed cell death in vitro, in situ and in vivo," Br. J. Cancer 81, 520-527 (1999).
8. F. L. Lizzi, M. Ostromogilsky, E. J. Feleppa, M. C. Rorke, and M. M. Yaremko, "Relationship of ultrasonic spectral parameters to features of tissue microstructure," IEEE Trans. Ultrason. Ferroelectr. Freq. Control UFFC-33(3), 319-329 (1986).

9. M. C. Kolios, G. J. Czarnota, M. Lee, J. W. Hunt, and M. D. Sherar, "Ultrasonic spectral parameter characterization of apoptosis," Ultrasound Med. Biol. 28, 589-597 (2002).

10. A. S. Tunis, G. J. Czarnota, A. Giles, M. D. Sherar, J. W. Hunt, and M. C. Kolios, "Monitoring structural changes in cells with highfrequency ultrasound signal statistics," Ultrasound Med. Biol. 31, 10411049 (2005).

11. V. Backman, M. B. Wallace, L. T. Perelman, J. T. Arendt, R. Gurjar, M. G. Muller, Q. Zhang, G. Zonios, E. Kline, and T. McGillican, "Detection of preinvasive cancer cells," Nature 406, 35 (2000).

12. A. L. Oldenburg, C. Xu, and S. A. Boppart, "Spectroscopic optical coherence tomography and microscopy," IEEE J. Sel. Top. Quantum Electron. 13, 1629-1640 (2007).

13. F. J. van der Meer, D. J. Faber, M. C. Aalders, A. A. Poot, I. Vermes, and T. G. van Leeuwen, "Apoptosis- and necrosis-induced changes in light attenuation measured by optical coherence tomography," Lasers Med. Sci. 25, 259-267 (2010).

14. B. J. Vakoc, R. M. Lanning, J. A. Tyrrell, T. P. Padera, L. A. Bartlett, T. Stylianopoulos, L. L. Munn, G. J. Tearney, D. Fukumura, R. K. Jain, and B. E. Bouma, "Three-dimensional microscopy of the tumor microenvironment in vivo using optical frequency domain imaging," Nat. Med. 15, 1219-1223 (2009).

15. V. X. D. Yang, G. J. Czarnota, I. A. Vitkin, M. Kolios, M. Sherar, J. de Boer, B. Tromberg, and B. C. Wilson, "Ultrasound backscatter microscopy/spectroscopy and optical coherence (Doppler) tomography for mechanism-specific monitoring of photodynamic therapy in vivo and in vitro," in Proc. SPIE 4612, 128 (2002).

16. R. M. Vlad, N. M. Alajez, A. Giles, M. C. Kolios, and G. J. Czarnota, "Quantitative ultrasound characterization of cancer radiotherapy effects in vitro," Int. J. Radiat. Oncol., Biol., Phys. 72, 1236-1243 (2008).

17. G. J. Czarnota, M. C. Kolios, H. Vaziri, S. Benchimol, F. P. Ottensmeyer, M. D. Sherar, and J. W. Hunt, "Ultrasonic biomicroscopy of viable, dead and apoptotic cells," Ultrasound Med. Biol. 23, 961-965 (1997).

18. M. Karbowski and R. J. Youle, "Dynamics of mitochondrial morphology in healthy cells and during apoptosis," Cell Death Differ 10, 870-880 (2003).

19. S. L. Fink and B. T. Cookson, "Apoptosis, pyroptosis, and necrosis: mechanistic description of dead and dying eukaryotic cells," Infect. Immun. 73, 1907 (2005).

20. G. Majno and I. Joris, "Apoptosis, oncosis, and necrosis. An overview of cell death," Am. J. Pathol. 146(1), 3-15 (1995).

21. D. B. Zamble and S. J. Lippard, "Cisplatin and DNA repair in cancer chemotherapy," Trends Biochem. Sci. 20, 435-439 (1995).

22. P. Dustin, "Microtubules," Sci. Am. 243, 66-76 (1980).

23. P. M. Shankar, "Ultrasonic tissue characterization using a generalized Nakagami model," IEEE Trans. Ultrason. Ferroelectr. Freq. Control 48, 1716-1720 (2001).

24. J. M. Schmitt, A. Knuttel, M. Yadlowsky, and M. A. Eckhaus, "Optical-coherence tomography of a dense tissue: statistics of attenuation and backscattering," Phys. Med. Biol. 39, 1705-1720 (1994). 